\title{
The Nosocomial Risk of Bacterial Contamination of Surgical Wards at Saint Francis Hospital in Katete District of Zambia
}

\author{
Frank Mtonga $^{1}$, Sydney Malama ${ }^{2 *}$, Geoffrey Kwenda ${ }^{1}$, Henry Chimana ${ }^{1}, J^{2}$ nn Bwalya Muma ${ }^{3}$ \\ ${ }^{I}$ Department of Biomedical Sciences, School of Health Sciences, University of Zambia, P. O. Box 50110, \\ Lusaka, Zambia \\ ${ }^{2}$ Health Promotions Research Program, Institute of Economic and Social Research, University of Zambia, P. O. \\ Box 30900, Lusaka, Zambia \\ ${ }^{3}$ Department of Disease Control, School of Veterinary Medicine, University of Zambia, P. O. Box 32379, \\ Lusaka, Zambia
}

*Corresponding Author: Sydney Malama, Health Promotions Research Program, Institute of Economic and Social Research, University of Zambia, P. O. Box 30900, Lusaka, Zambia, Email: sydneymalama19 $71 @$ gmail.com

\begin{abstract}
The World Health Organization (WHO) describes nosocomial infections as major infectious diseases having a huge economic impact despite the interventions being made to control the infections. This was a prospective study in which a total of 96 swabs were collected from the two surgical wards at St. Francis Hospital. Proportionately, of the potential pathogenic microorganism's contamination, S. aureus had $25.9 \%$, E. coli $14.8 \%$, P. species $14.8 \%$, P. aerugnosa $11.1 \%$, Enterobacter $11.7 \%$, S. pneumonia $7.4 \%$, Actinobacter species $7.4 \%$, Plesiomonas species 3.7\%, K. pneumonia 3.7\%, Hamophilus species $3.7 \%$. Further, most of the isolates exhibited highest resistance to penicillin (88.9\%), Cotrimoxazole and Ampicillin 66.7\%, Chloramphenicol 44.4\%, Clindamycin, $55.6 \%$ while others were below $20 \%$ with the lowest being Gentamycin and Tetracycline at $11.1 \%$ and Ceftazidime had a zero resistance (100\% sensitive). The surgical wards at St Francis hospital generally have high potential pathogenic microbial contamination, especially on the sinks, shower rooms and the nursing tables. Its therefore, imperative that the surrounding surfaces in the wards are routinely disinfected and sterilized to avoid cross contamination of both patients and medical personnel.
\end{abstract}

Keywords: Nosocomial, Katete, Surgical wards, Bacterial

\section{INTRODUCTION}

A surgical ward is a special unit within a hospital which admits patients who have undergone surgery or those waiting to undergo surgery and also other patients such as those with burns, injuries and dislocations that may not need surgery [1]. Reducing the health care associated infections in these wards remains a critical issue for clinicians and managers in hospitals and health care institutions all over the world [2]. According to the infection prevention control policy, very few surgical wound infections are acquired after the operation if there is primary closure of the wound. Open wounds and presence of drains increase the risk of infection in the post-operative period [3]. Various microbial communities have been uncovered in a variety of built environment and surprisingly from sites engineered to be sterile and high risk hospital wards [4,5].

The World Health Organisation (WHO) describes nosocomial infections as a major infectious diseases having a huge economic impact despite the interventions being made to control the infections [6]. It is estimated that surgical site infections develop in 2 to $5 \%$ of the 16 million people undergoing surgical procedure annually and account for $24 \%$ of all nosocomial infections [7]. Common Gram negative organisms isolated from infected surgical wounds include: Escherichia coli $(E$. coli), Klebsiella pnumoniae (K. pneumoniae), Pseudomonas arugenosa ( $P$. auregenosa), Proteus vulgaris ( $P$. vulgaris) and many others [8]. Staphylococcus aureus is the most common Gram positive microorganism isolated from 
surgical wounds though, others like coagulase negative bacteria, Staphylococcus aureus is also common since it is also found on the skin [9].

Microorganisms commonly isolated from septic surgical wounds are mostly acquired from patient-to-patient, hospital staff-to-patient and vice versa and from the hospital built in environment [10]. These organisms easily survive indoor environment because certain conditions support their survival. These include wet surfaces, warm room temperature, no direct exposure to the sun and many others [11]. The uncontrolled visits in patient wards also promote the continuation of contamination of the wards by different microorganisms. Not all postoperative wounds become infected because there are risk factors that predispose the wound to be infected by microorganisms and some of them include: low immunity, invasive medical procedure, infection at other sites, poor infection control practices, long stay in hospital and the age of the patient [6].

The chain of surgical wounds infection by microorganisms can be terminated by following simple instructions prior to and after carrying out a surgical operation. These include: decontamination of a patient's skin to preventing infection following a caesarean section; pre-operative bathing with skin antiseptics; antibiotics prophylaxis for surgery for proximal femoral and other closed long bone fractures; antibiotics prophylaxis for percutaneous endoscopic gastrostomy and changing behaviour to prevent [6].

There are scanty documented studies that have been done in Zambia with regard to surgical and hospital bacterial contamination. Therefore, this study was undertaken to ascertain the level of bacterial contamination in the male and female wards at St Francis Hospital, one of the level two and referral hospitals located in the eastern part of Zambia to ascertain the potential of nosocomial risk.

\section{Materials ANd Methods}

\subsection{Study Design}

This was a Prospective Study

\subsection{Study Site}

The study was conducted at St Francis Hospital from the male and female surgical wards. St
Francis Hospital is located in Katete district, eastern part of Zambia (Figure 1).

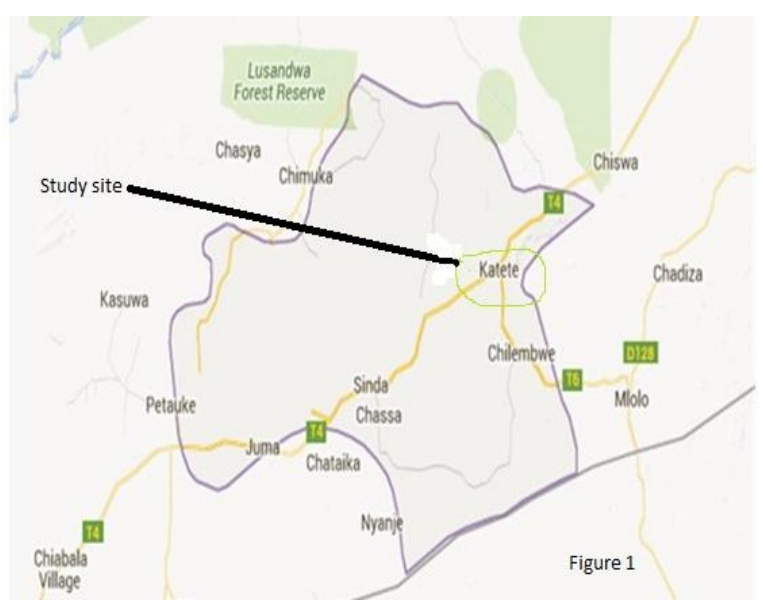

Figure1. Map showing the location of Katete district

The Hospital is a level two and serves as a referral centre for patients from the surrounding health facilities requiring specialised attention.

\subsection{Sample Collection}

All the surfaces and equipment in the Male and Female wards (sinks, nursing tables, toilet pans, showers, beddings, wall and dressing tables) were sampled using sterile moistened swabs. The samples were labelled, packed in Peptone containing broth and transported to the laboratory at the University teaching Hospital Microbiology laboratory for further analysis.

\subsection{Laboratory analysis}

The swabs collected were directly inoculated on blood agar, MacConkey agar, Chocolate agar. Blood agar and MacConkey agar plates were incubated aerobically at $37{ }^{\circ} \mathrm{C}$ while cultures on Chocolate agar plates were at anaerobic with $3 \%$ carbon dioxide for 24 hours. Positive culture growths were examined for colony characteristics, Gram stain reaction and biochemical characteristics as described by [9]. The biochemical characteristics tested were: Catalase, oxidase, coagulase, haemolysis, hydrogen sulphide production, citrate, TSI and indole, after identification sensitivities were set using the Muller Hinton agar at aerobic condition for 24 hours at $37{ }^{\circ} \mathrm{C}$. The antibiotics used included Gentamycin, chloramphenicol, Cotrimoxazole, Norfloxacin, ampicillin, penicillin,cefotaxime, cefoxitin, cefotriaxone, cefotriaxime, clindamycin, ciplofloxacin, erythromycin and tetracycline. The Kirby-baur method was employed and the processes was done as described by $[9,12]$. The choice of the antibiotics was based on the 
types of the isolates and their availability at $\mathrm{St}$ Francis hospital and Ridgeway microbiology laboratory.

\subsection{Data Analysis}

The excel analysis tool package was used to analyse the data. Data was entered in $\operatorname{Excel}^{\circledR}$ and analysed using the excel t-test/CORREL function (2010).

\subsection{Ethical Consideration}

Ethical clearance was sought from the University of Zambia School of Medicine Ethical Committee. Authorisation to conduct the study was sort from St Francis Hospital administration. The information obtained from this study was strictly used for research purposes.

\section{RESUlts}

A total of 96 swabs were collected from the two surgical wards at St. Francis Hospital. The number of swabs differed according to the anticipated contaminations and their significance in infection transmission in the wards. In their order, 20 swabs were collected from beddings with patients, 20 from showers, 15 from sinks, 15 from beddings without patients, 15 from nursing tables and 6 from selected wall sites. By percentage of the potential pathogenic microorganism's contamination, S. aureus had $25.9 \%$, E. coli $14.8 \%, \quad P$. species $14.8 \%$, Pseudomonas aerugnosa $11.1 \%$, Enterobacter $11.7 \%, S$. pneumonia $7.4 \%$, Actinobacter species $7.4 \%$, Plesiomonas species $3.7 \%$, K. pneumonia $3.7 \%$, Hamophilus species $3.7 \%$. Most of the isolates exhibited highest resistance to penicillin (88.9\%), Cotrimoxazole and Ampicillin 66.7\%, Chloramphenicol $44.4 \%$, Clindamycin, $55.6 \%$ while others were below $20 \%$ with the lowest being Gentamycin and Tetracycline at $11.1 \%$ and Ceftazidime had a zero resistance $(100 \%$ sensitive).

\section{DISCUSSION}

The findings in this study has indicated that the surgical environment at St Francis Hospital harbours potential pathogenic microorganisms which may cause infection, especially to immunocompromised patients if the infection prevention practices are not strictly adhered to. $S$. aureus and Gram negative bacilli were the most isolated microorganisms from the surfaces.
Generally, the sinks, the nursing tables and beddings with patients, showers, toilet pans in both wards had high percentages of contamination of which Staphylococcus spp, E. coli and Pseudomonas spp had the highest frequencies.

Staphylococcus aureus rated $25.9 \%$ and was isolated from most of the surfaces assessed in the surgical wards. This organism is found in most of the surfaces, especially where there is not much adherence to the day to day cleaning and disinfection. Most of the developing nations still have a challenge pertaining to this organism with regard to nosocomial infections. This observation is in agreement with the studies elsewhere [7,13] where Staphylococcus aureus prevalence in the surgical ward rated $23.8 \%$. In a related study, contamination of mattresses and blankets with Staphylococcus aureus in surgical wards under non epidemic conditions was reported [14]. This may indicate that in such circumstances, the disinfection policy is not fully enforced at the institution. In this study, ward attendants reported that disinfection was done twice or once in a day and while others doubted the frequency at which disinfection was done.

Pseudomonas isolation frequency rated $14.81 \%$ of the contamination levels. This organism can survive in hash conditions in the environment. Strict and well adhered attitude to disinfection plays a big role in controlling the organism. The organism was isolated from sinks and the showers. These are potential areas for cross infection of this pathogen either direct to the resident patient, health worker or to the the care taker of the patient [15]. In a recent study done at the UTH, Lusaka, Zambia, of the 107 white coats screened, $94(72.8 \%)$ were contaminated with bacteria [21].

E. coli and Proteus species rated 11.1\%, Streptococcus species and Actinobacter had 7.4\%, Enterobacter, Plesiomonas species, and Haemophilus species rated $3.07 \%$. Most of these pathogens found on the critical surfaces can easily be controlled with a well followed disinfection and general cleaning programme [16]. It has been demonstrated that microorganisms can survive between 10 and 98 days on fabrics found in hospitals, such as those used for coats as well as cotton and polyester materials [17]. 
As for the susceptibility antibiotic testing in the result section, the findings are in agreement with what has been observed elsewhere [5,18,19]. The results show that there is high chance of experiencing treatment failure from infections resulting from these bacteria.

This study has generally observed a high level of microbial resistance to antibiotics which has become a major public concern worldwide [6]. This may have been facilitated by the misuse of antibiotics in the community and hospital setups, poor infection control practices, inadequate sanitary conditions and inappropriate food handling [19].

\section{CONCLUSION}

The surgical wards at St Francis hospital generally have high potential pathogenic microbial contamination, especially on the sinks, shower rooms and the nursing tables. Its therefore, imperative that the surrounding surfaces in the wards are routinely disinfected and sterilized to avoid cross contamination of both patients and medical personnel.

\section{REFERENCES}

[1] Smith BA: Reducing surgical site infections: A patient care and business focus. OR Nurse 2015 2015, 9: 14-17.

[2] Maypole J, Sadof MD, Augustyn M: Medically Complex Care: The Newest Competency for Primary Care? Journal of Developmental \& Behavioral Pediatrics 2015, 36: 469-470.

[3] Curtis V, Aunger R, Rabie T: Evidence that disgust evolved to protect from risk of disease. Proceedings of the Royal Society of London B: Biological Sciences 2004, 271: S131-S133.

[4] Ghaferi AA, Birkmeyer JD, Dimick JB: Variation in hospital mortality associated with inpatient surgery. N Engl J Med 2009, 361: 1368-1375.

[5] Lee N, Lee H, Ko N, Chang C, HsinGÇÉI Shih $\mathrm{MD}, \mathrm{Wu} \mathrm{C}$ et al.: Clinical and economic impact of multidrug resistance in nosocomial Acineto bacter baumannii bacteremia. Infection control and hospital epidemiology 2007, 28: 713-719.

[6] Pittet D, Allegranzi B, Boyce J, World Health Organization World Alliance for Patient Safety First Global Patient Safety Challenge Core Group of Experts: The World Health Organization guidelines on hand hygiene in health care and their consensus recommendations. World Health 2009, 30: 611622.
[7] Ahmed MI: Prevalence of nosocomial wound infection among postoperative patients and antibiotics patterns at teaching hospital in Sudan. North American journal of medical sciences 2012, 4: 29.

[8] Fang G, Keys TF, Gentry LO, Harris AA, Rivera N, Getz $\mathrm{K}$ et al.: Prosthetic valve endocarditis resulting from nosocomial bacteremia: a prospective, multicenter study. Annals of internal medicine 1993, 119: 560567.

[9] Cheesbrough M: District laboratory practice in tropical countries. Cambridge university press; 2006.

[10] Boucher HW, Talbot GH, Bradley JS, Edwards JE, Gilbert D, Rice LB et al.: Bad bugs, no drugs: no ESKAPE! An update from the Infectious Diseases Society of America. Clinical Infectious Diseases 2009, 48: 1-12.

[11] Pittet D, Donaldson L: Clean Care is Safer Care: a worldwide priority. Lancet 2005, 366: 1246-1247.

[12] Isenberg HD: Mycobacteriology and antimycobacterial suscptibility testing. In Essential procedures for Clinical Microbiology. 1998 edition. Edited by Isenberg HD, Lynne SG. Washington, DC: American society for Microbiology Press; 1998:171-202.

[13] Arthur TD, Cavera VL, Chikindas ML: On bacteriocin delivery systems and potential applications. Future microbiology 2014, 9: 235248.

[14] Pinon A, Gachet J, Alexandre V, Decherf S, Vialette Ml: Microbiological Contamination of Bed Linen and Staff Uniforms in a Hospital. Advances in Microbiology 2013, 2013.

[15] Stein RA: Bacterial Infections of Humans: Epidemiology and Control. JAMA 2011, 305: 1488-1489.

[16] Jain A, Shah H, Jain A, Sharma M: Disinfection of stethoscopes: Gap between knowledge and practice in an Indian tertiary care hospital. Ann Trop Med Public Health 2013, 6: 236-239.

[17] Chacko L, Jose S, Isac A, Bhat KG: Survival of nosocomial bacteria on hospital fabrics. Indian journal of medical microbiology 2003, 21: 291.

[18] Boyce JM, White RL, Spruill EY: Impact of methicillin-resistant Staphylococcus aureus on the incidence of nosocomial staphylococcal infections. The Journal of infectious diseases 1983, 148: 763.

[19] Muto CA, Jernigan JA, Ostrowsky BE, Richet HM, Jarvis WR, Boyce JM et al.: SHEA guideline for preventing nosocomial 
transmission of multidrug-resistant strains of Staphylococcus aureus and enterococcus. Infection control and hospital epidemiology 2003, 24: 362-386.

[20] Saloojee H, Steenhoff A: The health professional's role in preventing nosocomial infections. Postgraduate medical journal 2001, 77: 16-19.
[21] Mwamungule S, Chimana HM, Malama S, Mainda G, Kwenda $G$ and Muma JB. Contamination of health care workers' coats at the University Teaching Hospital in Lusaka, Zambia: the nosocomial risk. Journal of Occupational Medicine and Toxicology (2015) 10:34

Citation: Frank Mtonga, Sydney Malama, Geoffrey Kwenda, Henry Chimana, John Bwalya Muma. The Nosocomial Risk of Bacterial Contamination of Surgical Wards at Saint Francis Hospital in Katete District of Zambia. ARC Journal of Clinical Case Reports. 2017; 3(4):14-18. doi:dx.doi.org/ 10.20431/24559806.0304004.

Copyright: (C) 2017 Authors. This is an open-access article distributed under the terms of the Creative Commons Attribution License, which permits unrestricted use, distribution, and reproduction in any medium, provided the original author and source are credited. 